\title{
Supporting Information for Detection of Sequence-Specific Protein-DNA Interactions via Surface Enhanced Resonance Raman Scattering
}

Andrew J. Bonham, Gary Braun, Ioana Pavel, Martin Moskovits, and Norbert O. Reich*

\section{Supporting Methods}

Oligonucleotide Preparation:

All oligonucleotides were synthesized using standard phosphoramidite chemistry and gel purified (Midland Certified Reagents). Oligonucleotide length and features were designed using previously established principles ${ }^{1}$. Disulfide groups were incorporated with standard reagents at the 5 ' or 3 ' termini of the oligonucleotide to provide a means of attachment for the oligonucleotides to the nanoparticle surface. Oligo-A and oligo-B contain 5' or 3' terminal disulfide couplings to allow them to bind gold surfaces, as well as ten-thymine spacer regions. Oligo-A and oligo-B were suspended at $400 \mu \mathrm{M}$ in $0.01 \mathrm{M}$ $\mathrm{NaH}_{2} \mathrm{PO}_{4} / \mathrm{Na}_{2} \mathrm{HPO}_{4}$ buffer, pH 7.5 (PB).

NP-Functionalization:

Gold NPs were created at a size regime that has been extensively explored under conditions of oligonucleotide functionalization ${ }^{1}$. To remove excess thiol-DNA, the gold NP solution was centrifuged until the NP formed an oily precipitate. After removal of the aqueous supernatant, the NP precipitate was washed twice by re-suspension in $0.1 \mathrm{M}$ $\mathrm{NaCl}, 0.01 \mathrm{M}$ PB followed by re-centrifugation, as above.

\footnotetext{
${ }^{1}$ J. Am. Chem. Soc., 125 (6), $1643-1654,2003$.
} 
The stability of these functionalized NPs in PBS (6 mM phosphate, $137 \mathrm{mM} \mathrm{NaCl}, 2.7$ $\mathrm{mM} \mathrm{KCl}, \mathrm{pH}$ 7.4) was similar for preparations made using free thiol or disulfide oligonucleotide attachment (data not shown), and in this study protein-DNA binding experiments were performed using unreduced disulfide oligonucleotides. Briefly, NPs were incubated in oligo-A or oligo-B for 24 hours at room temperature $(3 \mu \mathrm{M}$ final concentration of oligonucleotides), then aged in $0.1 \mathrm{M} \mathrm{NaCl}$ and $0.01 \mathrm{M}$ sodium phosphate buffer, $\mathrm{pH} 7.4$ for 48 hours and washed by centrifugation and re-suspended in PBS. The molar concentrations of NPs were determined by UV/Vis spectroscopy (molar extinction coefficient of $2.3 \times 10^{8}$ at $525 \mathrm{~nm}^{2}$, and the DNA functionalized NPs (NP-A and NP-B) were brought to and stored at a final concentration of $5 \mathrm{nM}$. TEM imaging and object analysis (UTHSCSA Image Tool) of the nanoparticles used in this study confirmed a diameter of $13.6 \pm 0.2 \mathrm{~nm}$ in the batch preparation.

The surface coverage of the oligonucleotides on the NPs was quantitated through absorbance measurements of the solution following the release of oligo-A (containing a fluorescein modification on the 5 , terminus) after displacement by $\beta$-mercaptoethanol ${ }^{3}$. NP-A conjugates were determined to have $183 \pm 20$ oligonucleotides per particle (Figure S2), giving an effective final DNA concentration in the colloidal suspension of $0.9 \pm 0.1$ $\mu \mathrm{M}$.

\footnotetext{
${ }^{2}$ Sung Yong Park; D.Stroud Physical Review B 2003, 68, 224201.

${ }^{3}$ Linette M.Demers; Chad A.Mirkin; Robert C.Mucic; Robert A.Reynolds; Robert L.Letsinger; Robert Elghanian; Garimella Viswanadham Analytical Chemistry 2000, 72, 5535-5541.
} 


\section{Proteins:}

Streptavidin was purchased from Leinco Technologies and suspended in PBS. M.HhaI was expressed and purified as described previously ${ }^{4}$. An E. coli construct for the expression of his-tagged yeast TBP was generously donated by Dr. Laurie Stargell (Colorado State University), expressed and purified as previously described ${ }^{5}$. Protein labeling with QSY21 was performed according to manufacturer protocols, and the labeled proteins were purified via two desalting column purifications with Bio-Gel P-6 Resin (Bio-Rad Laboratories). Extent of labeling was assessed using the proteins and labels mode of a NanoDrop spectrophotometer (NanoDrop).

\section{Assembly Formation:}

ABC assemblies were optimally formed in a step-wise process. The formation of assemblies causes characteristic shifts in the plasmon absorption maximum and can be monitored by UV-Vis spectroscopy (Figure S4). $10 \mu \mathrm{L}$ of NP-A were mixed with $10 \mu \mathrm{L}$ of NP-B and heated above the melting temperature of the oligonucleotide overhangs, 60 ${ }^{\circ} \mathrm{C}$ for 5 minutes. The temperature was then lowered to $45{ }^{\circ} \mathrm{C}$ at which point $\mathbf{C}$ was added to a final concentration of $1 \mu \mathrm{M}$. The solution was incubated at $45^{\circ} \mathrm{C}$ for 15 minutes and slowly cooled to room temperature. After 2 hours, a visible precipitation of NPs indicated the formation of $\mathrm{ABC}$ assemblies. To remove unbound $\mathbf{C}$, the solutions were centrifuged, the supernatant was removed, and the precipitate was re-suspended in PBS.

Protein incubation proceeded for 20 minutes at room temperature to allow equilibrium binding. The solutions were then gently centrifuged to palletize the assemblies.

\footnotetext{
${ }^{4}$ Zhou, H.; Shatz, W.; Purdy, M. M.; Fera, N.; Dahlquist, F. W.; Reich, N. O. Biochemistry 2007, 46, 72617268 .

${ }^{5}$ Stewart, J. J.; Stargell, L. A. J.Biol.Chem. 2001, 276, 30078-30084
} 
Supernatants containing non-bound protein were removed and discarded twice, and the assemblies were re-suspended in PBS. $3 \mu \mathrm{L}$ of each ABC assembly was drop-spotted onto a cleaned glass slide. Ag plating, wherein $\mathrm{AgNO}_{3}$ is chemically reduced and grows around the AuNPs, reduces the distance between analyte and metallic surface, leading to more intense SERS signals at the wavelengths used and significantly increases the number of intensely scattering "hot spots" observed ${ }^{6} .1 \mu \mathrm{L}$ of a Ag plating solution (LIS silver enhance, Nanoprobes) was applied and incubated for 6 minutes, followed by washing with $\mathrm{DI} \mathrm{H}_{2} \mathrm{O}$ and air-drying.

Transmission Electron Microscopy:

TEM imaging was performed on a JEOL JEM-1230 transmission electron microscope with a Hamamatsu Digital camera. Samples were deposited onto a copper-coated carbon grid (400 mesh, SPI), followed by drying. The TEM operating voltage was $80 \mathrm{kV}$ and the filament current was $56 \mathrm{uA}$.

\section{Raman Data Collection:}

Raman measurements were taken on a LabRam system (Horiba Jobin Yvon) equipped with 1200 grooves $/ \mathrm{mm}$ holographic gratings. The $633 \mathrm{~nm}$ output of a high power Melles Griot He-Ne laser was used to collect the spectra in the back-scattering geometry on a confocal Raman microscope (high stability BX40) equipped with Olympus objectives (LMPlanFl 100x, 50x, MPlan 10x). A Peltier CCD camera detection system was employed for the data acquisition. The spectral resolution was $1 \mathrm{~cm}^{-1}$. Typical measurements give a laser illumination spot of approximately $1 \mu \mathrm{m}$ diameter. Assemblies were identified via confocal microscopy. The laser intensity was set to $8.5 \mathrm{~mW}$, and the

\footnotetext{
${ }^{6}$ Cao, Y. C.; Jin, R.; Mirkin, C. A. Science 2002, 297, 1536-1540.
} 
Raman detector was calibrated using silicon. Sampling measurements were taken in line scanning mode with 5 second integration time on each line.

\section{Supporting Data}

When oligo $\mathrm{C}$ was present in sub-equimolar ratios compared to surface-bound oligo A or B, overnight incubation at room temperature was required for significant aggregation. When equimolar oligo $\mathrm{C}$ was used, aggregation was detectable after 4 hours incubation. When a large excess of oligo $\mathrm{C}$ was added ( $>10$ times the amount of either oligo $\mathrm{A}$ or $\mathrm{B})$, the NPs do not significantly assemble out of solution.

For functional binding, the DNA scaffolding of the $\mathrm{ABC}$ assemblies must remain sterically accessible for protein binding. To ensure this, surface coverage conditions of NPs with disulfide-terminated oligonucleotides was optimized. The surface coverage of the NPs by the oligonucleotide sequence used for hybridization was varied by the introduction of shorter 'diluent' DNA sequences (ten-thymine disulfide oligos) ${ }^{7}$. This poly-thymine sequence is unable to hybridize with the bridging oligo-C and should not be recognized by the DNA-binding proteins, but still provides a functionalized oligonucleotide shell around the NP to provide stability in solution and promotes an upright orientation from the surface. Diluent strands were mixed in varying ratios with the surface DNA oligos A and $\mathbf{B}$ and introduced to the NPs to create a mixed surface functional layer. When the diluent was present in a greater than 1:1 ratio compared to the longer, probe strand, NPs were not stable in PBS for greater than 24 hours. In contrast,

\footnotetext{
${ }^{7}$ Storhoff, J. J.; Lazarides, A. A.; Mucic, R.; Mirkin, C.; Letsinger, R.; Schatz, G. C. J.Am.Chem.Soc. 2000, 122, 4640-4650.
} 
NPs with a less than 1:1 ratio of diluent:probe or with no diluent oligos were stable in PBS for periods greater than three weeks.

The binding site, contained in oligo-C, may affect the extent to which DNA-binding proteins can assemble within these assemblies. To quantitate diluent effects on the number of binding sites per NP, $\beta$-mercaptoethanol stripping and absorbance measurements were performed on assemblies containing fluorescein-labeled oligo-C, and correlated with diluent:probe ratio. With no diluent present, $18 \pm 20$ oligo-C strands/particle were bound, whereas when diluent was present at a 1:1 diluent:probe ratio $36 \pm 11$ strands/particle were accessible (Figure S3). Despite this moderate difference, assemblies formed from both sets of AuNPs had similar assembly size and morphology. For all the protein-DNA binding studies we consequently used a non-diluent system.

NP-A either free or hybridized to oligo-C did not form assemblies (Figure S6, a or b). When NP-A and NP-B are hybridized together with oligo-C, aggregation was observed (Figure S6c). All assemblies could be returned to colloidal suspension by heating for 20 minutes at $80{ }^{\circ} \mathrm{C}$ (data not shown).

The affinity of the QSY-labeled recombinant TBP used in this study was tested using an electrophoretic mobility shift assay ${ }^{8}$ (Figure S5). The apparent dissociation constant of $4.4 \mathrm{nM} \pm 1.0 \mathrm{nM}$ correlates with literature reported values, and is similar to values obtained for unlabeled TBP. The activity of QSY-labeled M.HhaI was assessed via methylation burst assay ${ }^{9}$, and displayed a $\mathrm{k}_{\mathrm{cat}}$ of $0.01 \mathrm{sec}^{-1}$, 4-fold impacted from the

\footnotetext{
${ }^{8}$ Stewart, J.J. and L.A. Stargell. (2001). J. Biol. Chem. 276, 30078-84.

${ }^{9}$ William M. Lindstrom, Jr., James Flynn, and Norbert O. Reich J. Biol. Chem. 275: 4912-4919.
} 
wildtype value of $0.042 \mathrm{sec}^{-1}$. These assessments indicate that QSY21 labeling is not disrupting the ability to bind DNA or perform enzymatic functions for the proteins presented in this study. Further studies into alternative labeling strategies, such as through thiol-reactive dyes, may be desirable for general applicability to DNA-binding proteins.

Enhancement Factor:

To provide a rough objective measure of the enhancement effect of our assemblies, we used the parameter $\mathrm{AEF}^{10}$. AEF is defined as $A E F=I_{\text {SERS }} / I_{0} \times C_{0} / C_{\text {SERS }}$, where, $I_{\text {SERS }}$ refers to the intensity of a vibrational mode in the SERS spectra of QSY21 in our assemblies and $\mathrm{I}_{0}$ refers to the same mode of QSY21 in silver colloid solution, $\mathrm{C}_{\text {SERS }}$ is the concentration of QSY21 in our assemblies and $\mathrm{C}_{0}$ is the concentration of QSY21 in the silver colloid solution. The AEF was obtained by taking the ratio of the integrated peak area at $1641 \mathrm{~cm}^{-1}$ in the SERS spectrum from the lowest concentration data point for QSY-M.HhaI and the integrated peak area of QSY21 in silver colloid solution. Taking into account the different laser powers and integration times used to collect each spectrum, we calculated an AEF of $3.5 \times 10^{4}$. Integrated signal intensity for the $1641 \mathrm{~cm}^{-}$ ${ }^{1}$ peak for $20 \mathrm{nM}$ QSY-M.HhaI was 100 counts with a $5 \mathrm{~s}$ integration using line scanning mode at $17 \mathrm{~mW}$, giving an approximate power at each spot of, at most, $850 \mu \mathrm{W}$. The reference QSY21 spectrum was 1000 counts, collected in point mode using a power of $170 \mu \mathrm{W}$ and an integration time of 60 seconds. Although the AEF values do not reveal the full enhancement due to the "hot spot" formation in the assemblies, it demonstrates

\footnotetext{
${ }^{10}$ Yuling Wang, Hongjun Chen, Shaojun Dong and Erkang Wang. J. Raman Spectrosc. 2007; 38: 515-521.
} 
that the controlled assembly and silver plating provides a $10^{4}$ enhancement from incubation with silver colloid alone.

\section{DNA sequences used in this study}

\begin{tabular}{l|l} 
Name & Sequence \\
\hline Oligo-A & 5'-TCTCAACTCGTATTTTTTTTT-disulfide-3' \\
Oligo-B & 5'-disulfide-TTTTTTTTTTCGCATTCAGGAT-3' \\
Diluent & 5'-TTTTTTTTTT-disulfide-3' \\
$\begin{array}{l}\text { Oligo-C, HhaI, } \\
\text { top }\end{array}$ & 5'-TACGAGTTGAGAAGACGAGCGCATGCAATCCTGAATGCG-3' \\
$\begin{array}{l}\text { Oligo-C, HhaI, } \\
\text { bottom }\end{array}$ & 5'-TGCATGCGCTCGTCT-3' \\
$\begin{array}{l}\text { Oligo-C, TBP, } \\
\text { top }\end{array}$ & 5'-TACGAGTTGAGAAGACGAGCGCGGGGCTATAAAAGGGGGTGGCATGCAATCCTGAATGCG-3' \\
$\begin{array}{l}\text { Oligo-C, TBP, } \\
\text { bottom }\end{array}$ & 5'-TGCATGCCACCCCCTTTTATAGCCCCGCGCTCGTCT-3' \\
$\begin{array}{l}\text { Oligo-C, } \\
\text { streptavidin, top }\end{array}$ & 5'-TACGAGTTGAGAAGACGAGCGCATGCAATCCTGAATGCG-3' \\
$\begin{array}{l}\text { Oligo-C, } \\
\text { streptavidin, } \\
\text { bottom }\end{array}$ & 5'-TGCATGCGC-(Biotin-dT)-CGTCT-3' \\
&
\end{tabular}

\section{Supporting Figures}

\section{$\begin{array}{llllll}1 & 2 & 3 & 4 & 5 & 6\end{array}$}

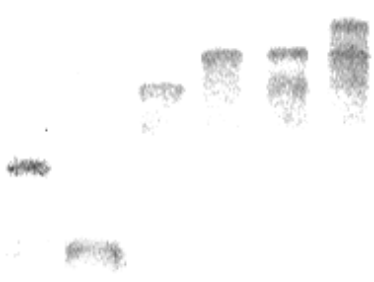

Figure S1: Annealing gel of the ABC DNA scaffolding assembly used in this study. Lanes 1 and 2 (contrast enhanced) show the top and bottom single-stranded DNA that hybridize to form oligo-C, lane 3. When oligo-C is hybridized to oligo-A (lane 4) or 
oligo-B (lane 5), a larger complex is observed. When oligo-A, oligo-C, and oligo-B are all hybridized, the full scaffolding forms (lane 6).

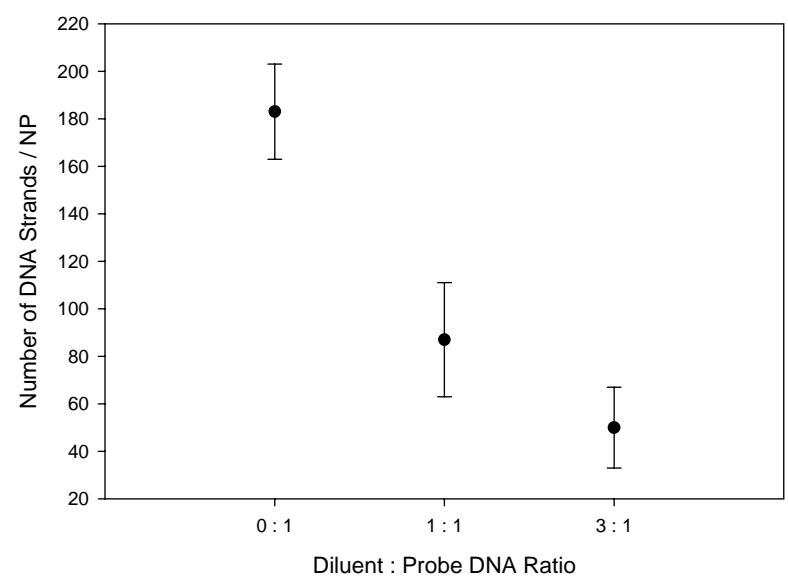

Figure S2: Surface coverage of fluorescently-labeled oligo-A varies with diluent:oligoA DNA strand ratio. AuNPs were functionalized with $0: 1$, 1:1, or $3: 1$ ratios of diluent:oligo-A DNA, then repeatedly washed to remove unbound DNA. Treatment with $\beta$-mercaptoethanol displaced the DNA from the AuNP surface (causing AuNP precipitation) and absorbance at $494 \mathrm{~nm}$ was measured for the solution, providing a measure of fluorescein concentration. These concentrations were compared to the measured AuNP concentration to generate a measure of fluorescent DNA strands per nanoparticle. The smaller size of the diluent strand compared to oligo-A may account for the greater apparent affinity of the diluent for the AuNP surface. 


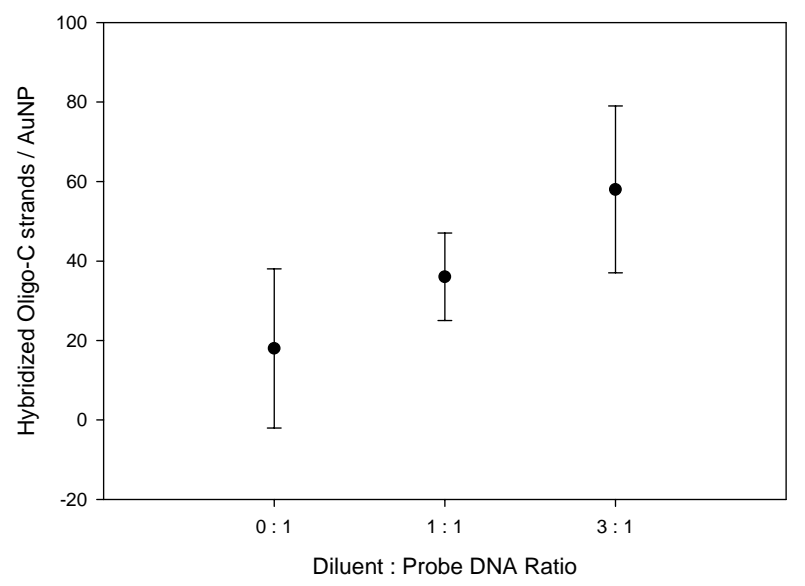

Figure S3: Accessibility of oligo-C hybridization in assemblies varies with diluent:probe DNA ratio on surface of AuNPs. AuNPs were functionalized with $0: 1,1: 1$, or $3: 1$ ratios of diluent:probe DNA, and fluorescently-labeled oligo-C was allowed to hybridize. After repeated washes to remove unhybridized oligo-C, DNA was displaced from AuNP by $\beta$ mercaptoethanol (causing AuNP precipitation) and absorbance at $494 \mathrm{~nm}$ was measured for the solution, providing a measure of fluorescein concentration. This was related to measured AuNP concentration in solution to generate fluorescent-strands per nanoparticle. Assemblies prepared with a 3:1 diluent:probe DNA ratio were not stable in solution over time.

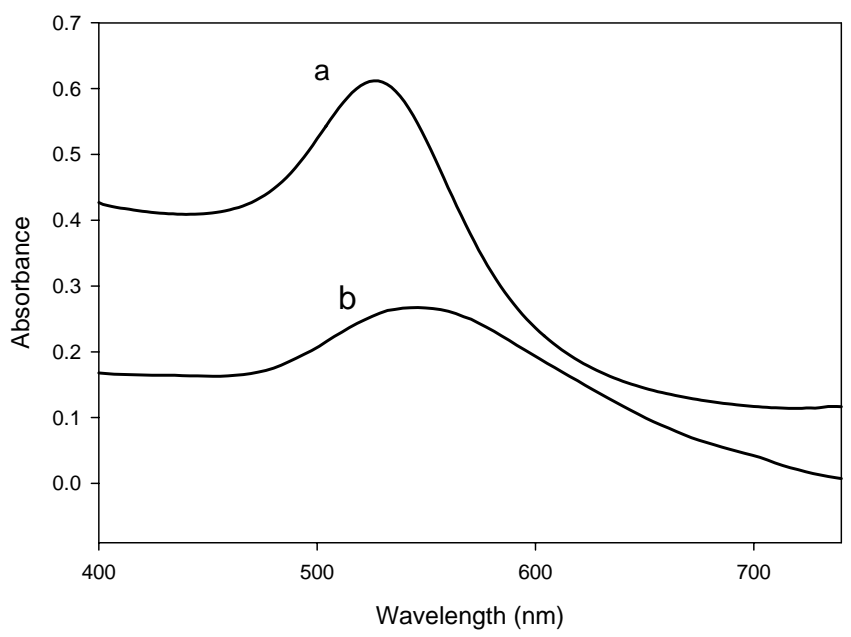


Figure S4: AuNPs are brought into close proximity upon DNA hybridization to form assemblies, resulting in a characteristic Plasmon resonance absorption shift. a, absorbance spectrum of DNA functionalized AuNP. b, absorbance spectrum after aggregation displays peak broadening and red-shift.

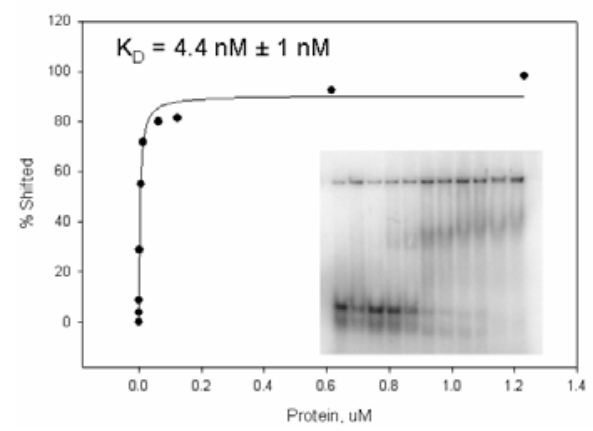

Figure S5: Binding isotherm of the His-tagged labeled-TBP used in this study. Apparent dissociation constant of $4.4 \mathrm{nM} \pm 1.0 \mathrm{nM}$ correlates with literature reported values. Gel shift was performed on a $8 \%$ acrylamide gel, $0.5 \mathrm{x}$ TBE with $5 \mathrm{mM} \mathrm{MgCl}_{2}$ at 25 degrees.
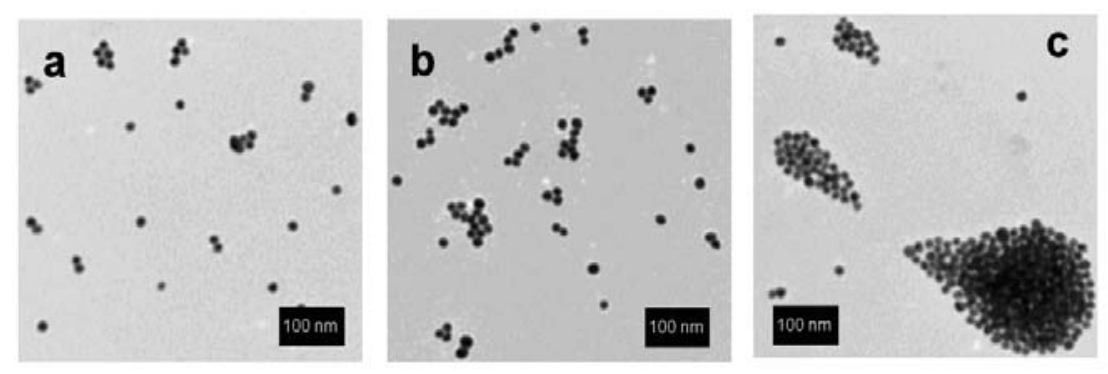

Figure S6: Bright field TEM images showing (a) NPs decorated with oligo A, (b) NPs decorated with oligo A and annealed with oligo C, and (c) the full system of NPs decorated with $\mathrm{A}$ and $\mathrm{B}$, formed into assemblies by the addition of oligo $\mathrm{C}$. 